Supplementary Information for

\title{
Interwall Friction and Sliding Behavior of Centimeters Long \\ Double-Walled Carbon Nanotubes
}

Rufan Zhang, Zhiyuan Ning, Ziwei Xu, Yingying Zhang, ${ }^{*}$ Huanhuan Xie, Feng Ding,

Qing Chen, Qiang Zhang, Weizhong Qian, Yi Cui, Fei Wei*

\section{Supplementary figures}

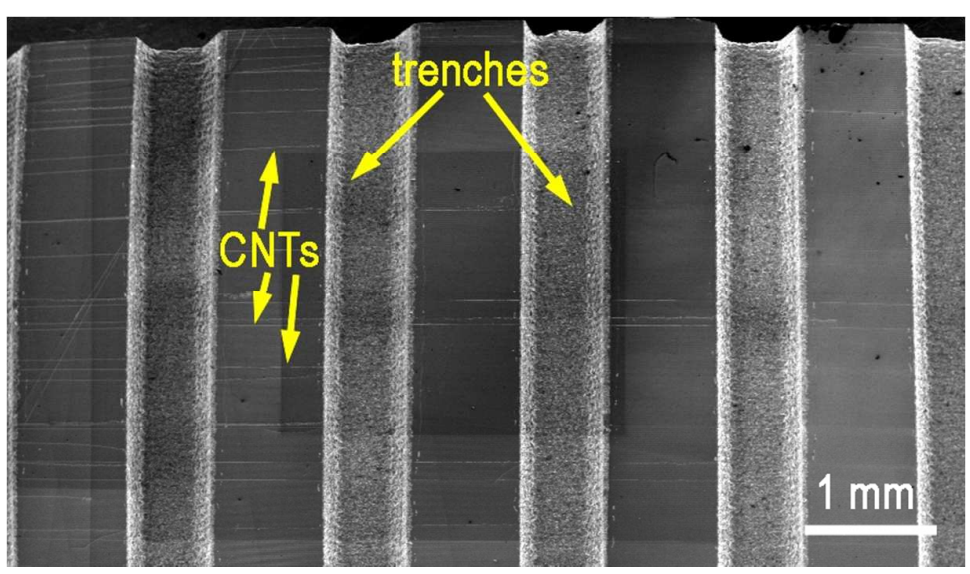

Figure S1. SEM image of ultralong CNTs grown on a silicon substrate with trenches on it.

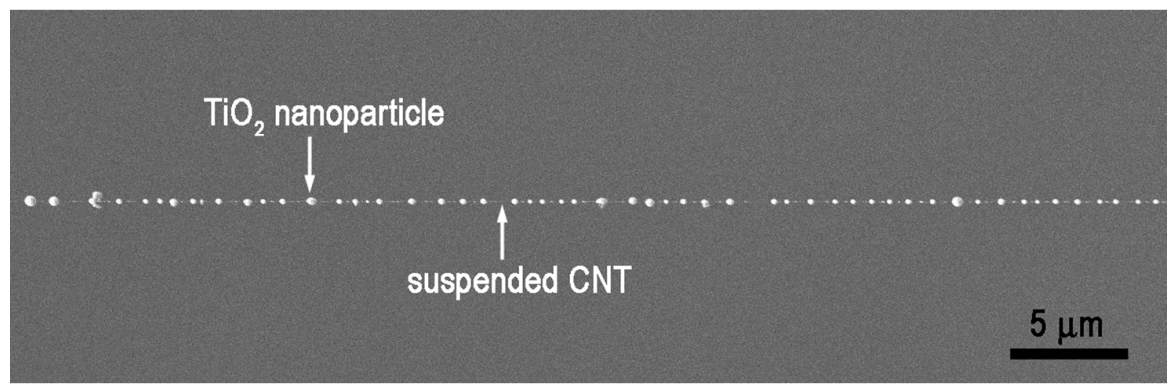

Figure S2. SEM image of suspended CNT decorated with $\mathrm{TiO}_{2}$ nanoparticles. 

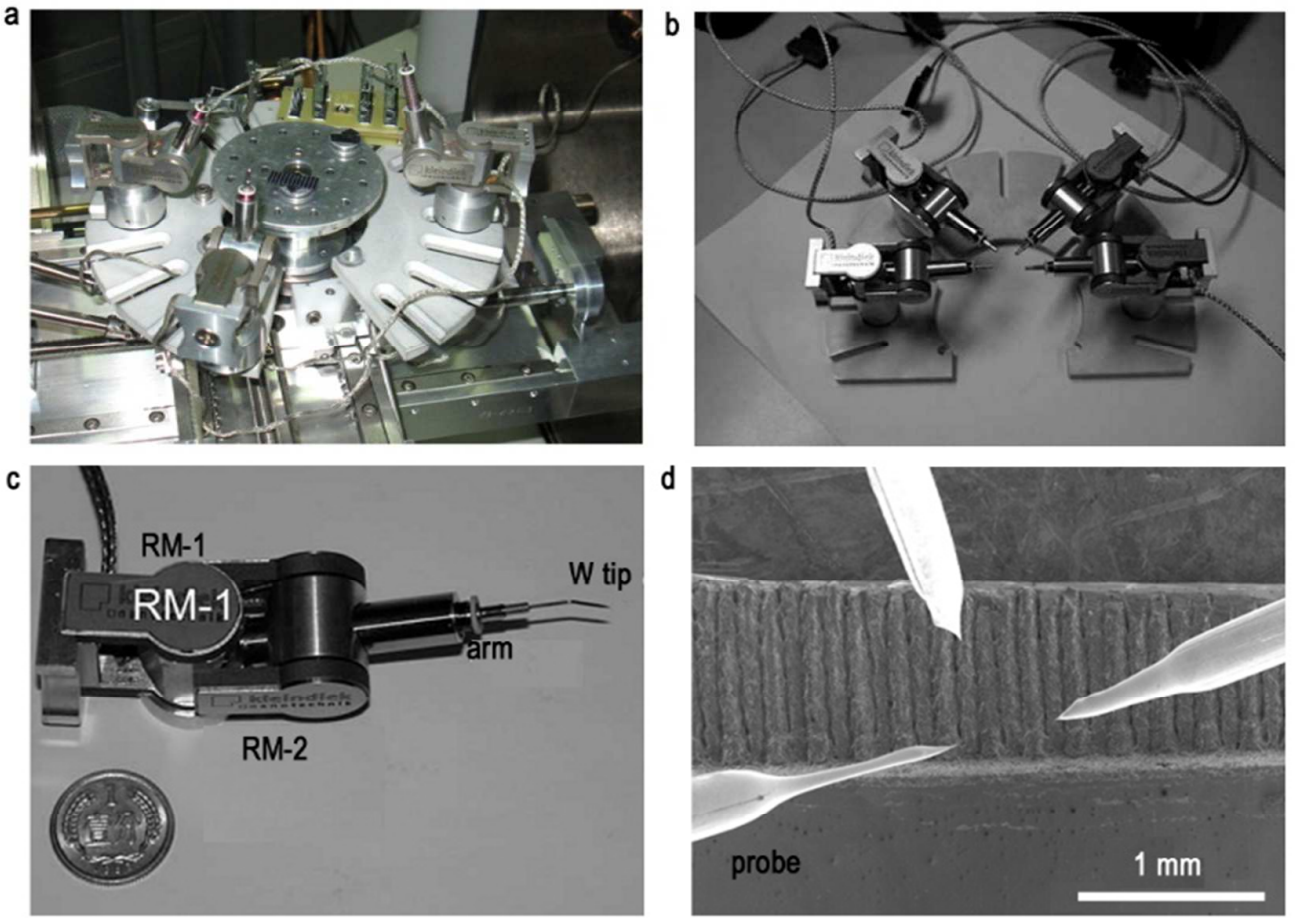

Figure S3. Experimental setup for manipulating individual CNTs. (a) An internal view of the manipulation station with four MM3A probes inside the SEM. The probes can be moved in three-dimensions with a precision of $0.25 \mathrm{~nm}$ per step. (b) Four MM3A probes were mounted on a probe cradle. (c) A MM3A nanoprobe. (d) SEM image of three $\mathrm{W}$ probes.

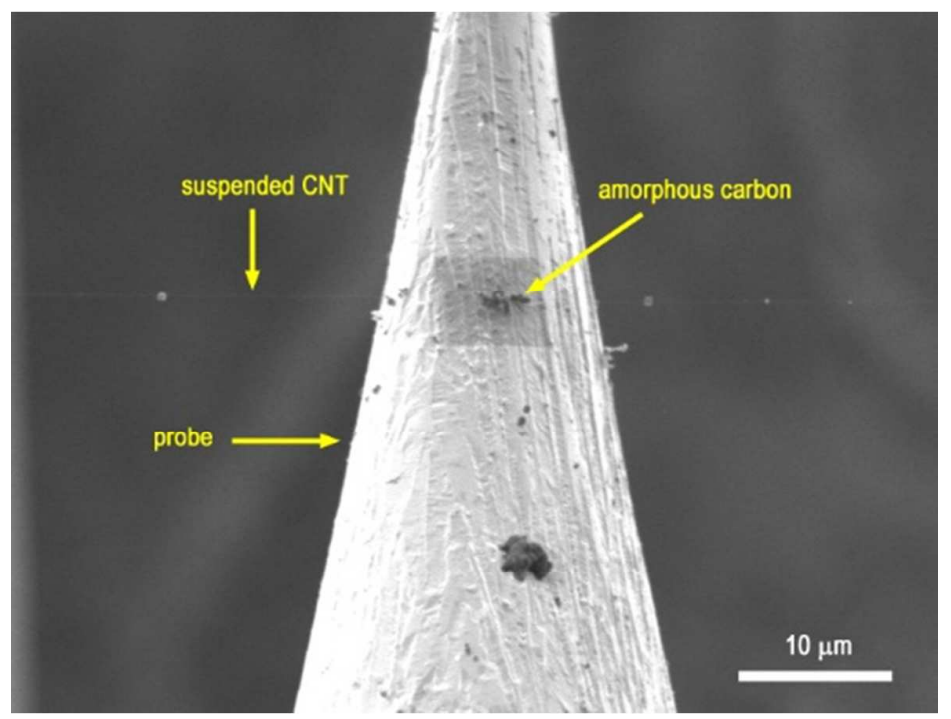

Figure S4. SEM image of fixing a suspended CNT onto a tungsten probe by 
depositing a layer of amorphous carbon on the contact area (the z-direction of the probe movement is perpendicular to the plane).

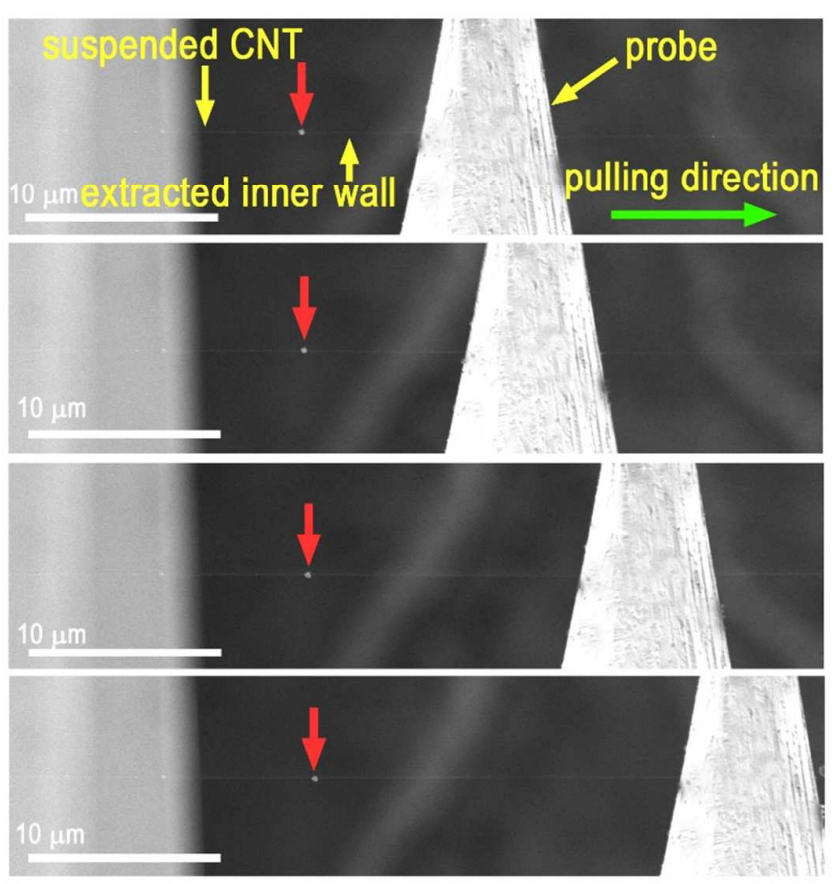

Figure S5. Process of pulling out the inner wall from the DWCNT with a probe.

The $\mathrm{TiO}_{2}$ particle shown by the red arrows was on the outer wall and kept unmoved during the pull-out process of the inner wall. The moving direction of the nanowire was from left to right. Illustration of the above processes was shown in the insets (the z-direction of the probe movement is perpendicular to the plane). 


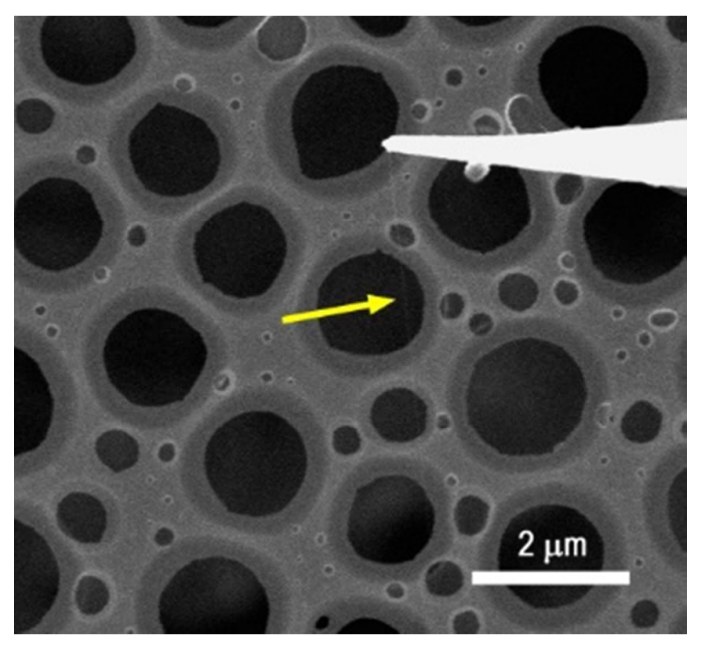

Figure S6. Transferring the extracted inner tube with a probe to the TEM grid. The yellow arrow indicates the extracted inner tube.

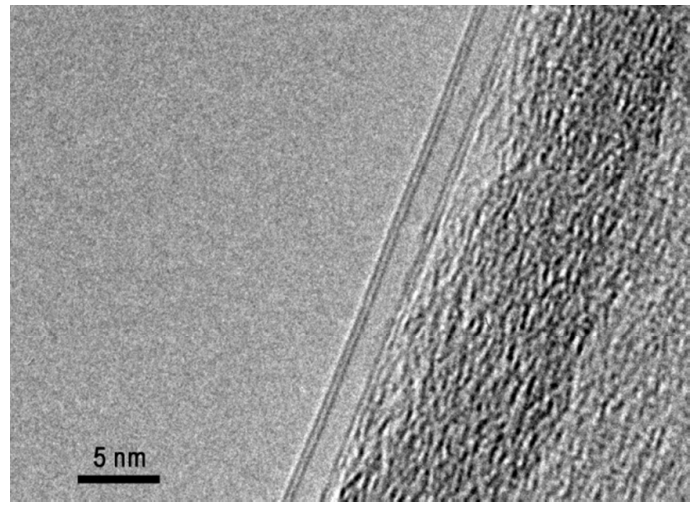

Figure S7. TEM image of a DWCNT

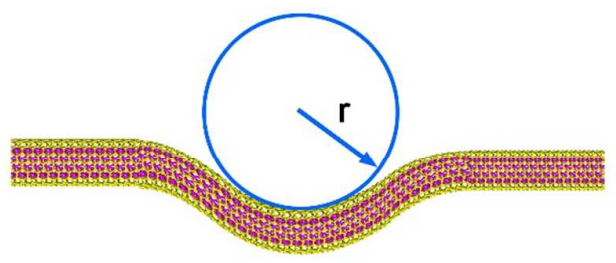

Figure S8. Illustration of curvature radius of a curved CNT. 

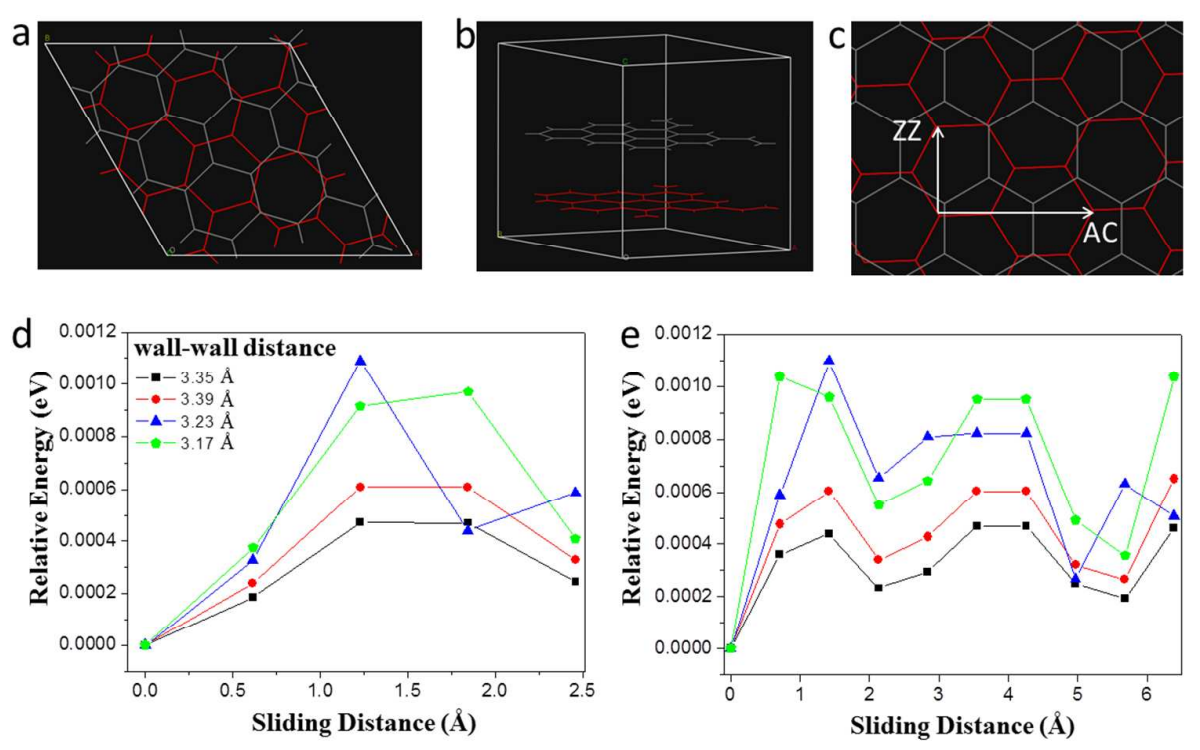

Figure S9. The variation of the relative van der Waals (vdw) energy vs. the sliding distance of an incommensurate bilayer graphene for different wall-wall distances. (a-b) Top and side views of the supercell of incommensurate bilayer graphene with the relative rotation angle $\theta=13.897^{\circ}$. (c) Two directions for the sliding of the one layer: zigzag (ZZ) direction and armchair (AC) direction. (d-e) The variations of the relative vdw energies as function of sliding distances for different wall-wall distances along the $\mathrm{ZZ}$ and $\mathrm{AC}$ directions, respectively. 